\title{
Stimmt das Volumen der Pipette?
}

\section{Antonio Romaguera}

Nahezu jedes Labor nutzt Kolbenhubpipetten mit Luftpolster, im allgemeinen Sprachgebrauch oft als

Mikroliterpipetten bezeichnet. Diese sind im Rahmen der Prüfmittelüberwachung regelmäßig zu

kalibrieren. Da Faktoren wie Luftfeuchtigkeit und Temperatur die Ergebnisse stark beeinflussen, sind

die Prüfbedingungen exakt einzuhalten. Worauf kommt es wirklich an?

- Laboratorien, die nach ISO 9001 zertifiziert, nach ISO 17025 akkreditiert sind oder nach den GLP/GMP-Richtlinien arbeiten, sind verpflichtet, ihre Pipetten im Rahmen der Prüfmittelüberwachung regelmäßig zu kalibrieren, denn diese sind qualitätsrelevante Messmittel. Die Kalibrierung dient dazu, Abweichungen des dosierten Volumens vom eingestellten Volumen zu ermitteln und mit den geforderten Fehlergrenzen für die systematische (Richtigkeit) und zufällige (Variationskoeffizient) Messabweichung zu vergleichen. Wie oft die Pipetten zu prüfen sind, hängt in Anlehnung an die ISO 10012 (Messmanagementsysteme Anforderungen an Messprozesse und Messmittel) unter anderem davon ab, wie oft und mit welchen Flüssigkeiten sie genutzt werden. Die meisten Pipettenhersteller empfehlen eine Überprüfung alle drei bis zwölf Monate.

Die eigentliche Kalibrierung von Kolbenhubpipetten beschreiben detailliert Teil 2 und Teil 6 der DIN EN ISO 8655. Goldstandard ist heute die gravimetrische Prüfung. Alternativen wie die photometrische Messung stehen in Teil 7 der Norm. In der Norm findet man Angaben zu den einzuhaltenden Umgebungsbedingungen, zur eigentlichen Durchführung der Prüfung und zur abschließenden Auswertung. ${ }^{1)}$

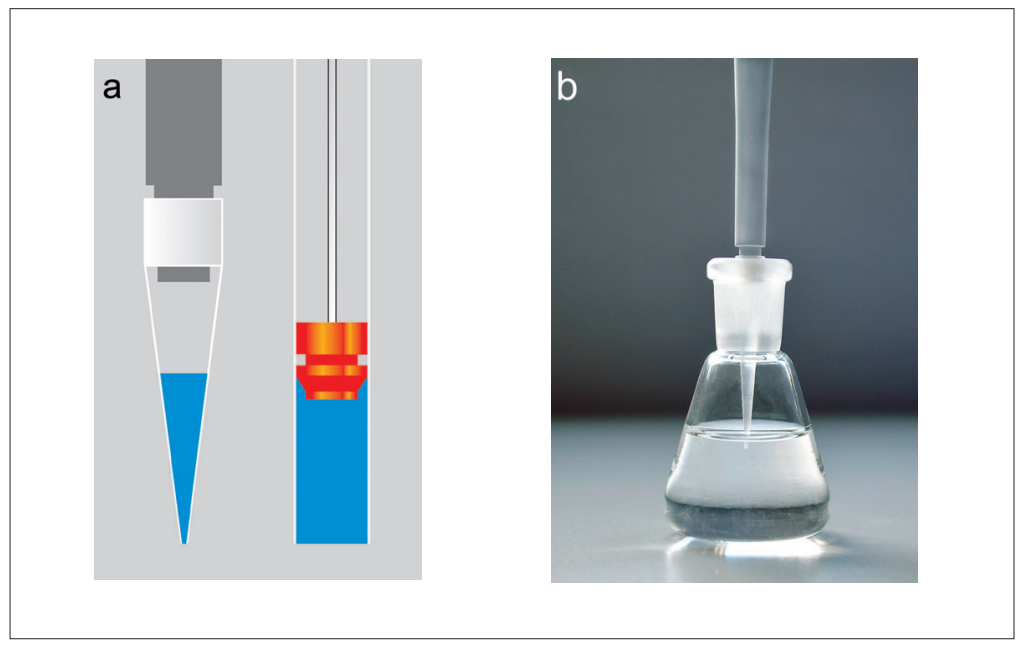

Abb. 1. a) Links: Luftpolsterpipette: Das Luftpolster zwischen Flüssigkeitsoberfläche und Kolben wird von den Umgebungsbedingungen beeinflusst; rechts: Direktverdrängerpipette. b) Eintauchtiefe und Haltung der Pipette beim Aufsaugen; beides beeinflusst die aufgenommene Flüssigkeitsmenge.

\section{Umgebungsbedingungen}

Aufgrund der Bauform und des Arbeitsprinzips von Luftpolsterpipetten (Abbildung $1 \mathrm{a}$, links) spielen die Umgebungsbedingungen bei der Kalibrierung eine weitaus größere Rolle als bei anderen Liquid-Handling-Geräten, zum Beispiel Handdispensern oder Flaschenaufsatzdispensern, die Direktverdränger sind. Schwankungen der Temperatur, der relativen Luftfeuchtigkeit und des Luftdruckes wirken sich direkt auf die Verhältnisse im Luftpolster aus und verursachen große Fehler. Ist beispielsweise die Luft im Prüfraum und somit auch im Luftpols- ter der Pipette zu Prüfbeginn trocken und saugt man die Prüfflüssigkeit auf, verdunstet über die Flüssigkeitsoberfläche das Wasser in das Luftpolster hinein. Dadurch vergrößert sich das Volumen des Luftpolsters, und das abgegebene Volumen ist zu niedrig.

Die Ergebnisse zahlreicher Untersuchungen führten Ende 2011 zur Erstellung und Veröffentlichung der DKD-Richtlinie 8-1 (DKD-R 8-1 „Kalibrierung von Kolbenhubpipetten mit Luftpolster" $)^{2)}$ und eines Berichts zu dieser Richtlinie („Analyse der Einflussgrößen auf die Kalibrierung von Kolbenhubpipetten mit Luftpolster"). ${ }^{3)}$ Beide Unterlagen 
können von der Homepage der Physikalisch-Technischen Bundesanstalt heruntergeladen werden. ${ }^{2,3)}$

\section{Der Einfluss von Temperatur und Luftfeuchtigkeit}

- Während der Kalibrierung verfälschen Temperaturdifferenzen zwischen dem destillierten Wasser, der Pipette und der Luft das Ergebnis. Für einen sicheren Temperaturausgleich müssen Luftpolsterpipetten mindestens zwei Stunden im klimatisierten Messraum gelagert werden.

Während der Kalibrierung sollte die Differenz zwischen der Raum- und der Wassertemperatur unterhalb von $0,5 \mathrm{~K}$ liegen, und auch die Temperatur der Raumluft darf maximal um $\pm 0,5 \mathrm{~K}$ schwanken.

Durch die Verdunstung der Prüfflüssigkeit (Wasser der Qualität 3 nach DIN ISO 3696) aus dem Vorratsgefäß kühlt sich diese ab, was bei den nachfolgenden Berechnungen $\mathrm{zu}$ berücksichtigen ist. Daher ist die Temperatur der Prüfflüssigkeit regelmäßig zu überwachen, und die Wasseroberfläche im Vorratsgefäß sollte möglichst klein sein.

Die DKD-Richtline 8-1 fordert eine relative Luftfeuchtigkeit von $45 \%$ bis $60 \%$ für die Kalibrierung von Pipetten. Wie beschrieben, führt eine niedrige Luftfeuchte zu einem verringerten gemessenen Volumen. Grundsätzlich ist die Verdunstung zusätzlich durch die Verwendung von Verdunstungsfallen, Mikrowägegefäßen und ähnlichem zu minimieren.

\section{Arbeitstechnik beim Kalibrieren: Volumen, Eintauchtiefe, Abgabe}

Kalibriert werden variable $\mathrm{Pi}$ petten bei $10 \%$, 50\% und $100 \%$ des Nennvolumens. Die Prüfung sollte bei $10 \%$ des Nennvolumens begonnen werden. Volumina unter $10 \%$ sind nicht zu prüfen.
In einem ersten Schritt muss das Gerät nach dem Aufstecken einer Pipettenspitze durch fünfmaliges Aufsaugen und Abgeben der Prüfflüssigkeit konditioniert werden. Das Vorkonditionieren reduziert das Verdunsten von Flüssigkeit in das Luftpolster hinein.

Nach ISO 8655 Teil 2 ist für jede Messung eine neue Pipettenspitze $z u$ benutzen, die zuvor einmal vorbefeuchtet wurde. Untersuchungen zeigen jedoch, dass ein Spitzenwechsel das zuvor erreichte Gleichgewicht im Luftpolster wieder zerstört. Das Ergebnis dieser Untersuchungen steht in der DKD-R 8-1. Das Arbeiten mit nur einer Spitze für einen Volumenbereich ist somit zulässig. Die Spitze sollte erst dann gewechselt werden, wenn das Volumen eingestellt ist. Auch dann ist ein fünfmaliges Vorbefeuchten notwendig.

Beim Aufsaugen der Flüssigkeit und Herausziehen der Spitze aus der Prüfflüssigkeit muss die $\mathrm{Pi}$ pette senkrecht stehen (Abbildung $1 \mathrm{~b}$ ). Wenn die Flüssigkeit wieder abgegeben wird, kann der Winkel $30^{\circ}$ bis $45^{\circ}$ betragen. Während des Aufsaugvorganges darf die Pipettenspitze die Gefäßwand nicht berühren.

Die Eintauchtiefe und die Wartezeit in der Prüfflüssigkeit hängen vom Volumenbereich der Pipette ab (Tabelle 1, S. 1142).

Nach dem Abstreifen der Spitze an der Gefäßwand darf sich die Spitzenöffnung beim Zurückziehen des Kolbens nicht zu nah am Meniskus der Flüssigkeit befinden, um die versehentliche Aufnahme von Flüssigkeit zu vermeiden.

Insgesamt sind zehn Messungen pro Prüfvolumen und Kanal durchzuführen. Die Kalibrierung von Pipetten mit einem Volumenbereich von $10 \mu \mathrm{L}$ bis $1 \mathrm{~mL}$ erfordert nach ISO 8655 eine fünfstellige Waage, die Kalibrierung von Pipetten mit einem Volumen unter $10 \mu \mathrm{L}$ verlangt eine Waage mit sechs Nachkommastellen (Tabelle 2, S. 1142).

Den Ablauf einer vollständigen Prüfung zeigt Abbildung 2.

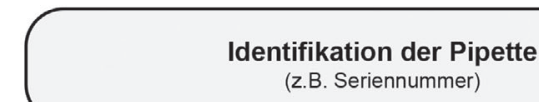

(z.B. Seriennummer)

Temperaturanpassung der Pipette (mindestens $2 \mathrm{~h}$ im Prüfraum)

Mindestanforderungen an Umgebungsbedingungen Lufttemperatur $20^{\circ} \mathrm{C}$ bis $25^{\circ} \mathrm{C}$

rel. Lufffeuchte $45 \%$ bis $60 \%$

Luftdruck muss bekannt sein

max. Temperaturdifferenz zwischen Wasser und Pipette $0,5^{\circ} \mathrm{C}$

Raumtemperatur darf während der Prüfung nur $+/-0,5^{\circ} \mathrm{C}$ schwanken

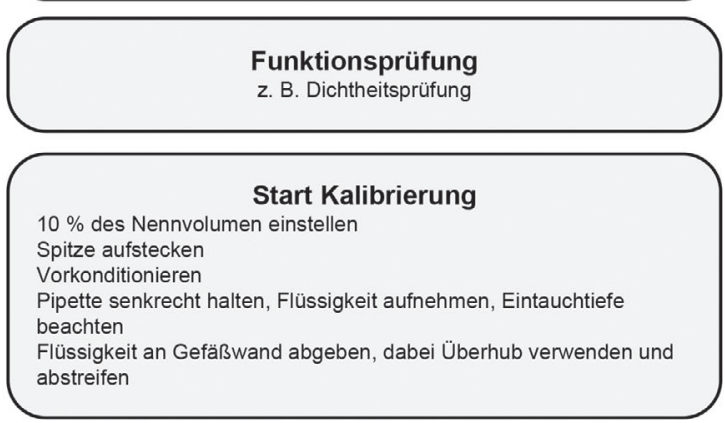

\section{Wägewert dokumentieren}

Vorgang 9 mal wiederholen

Spitze wechseln und erneut Vorkonditionieren

Vorgang mit $50 \%$ des Nennvolumens wiederholen

Vorgang mit Nennvolumen wiederholen

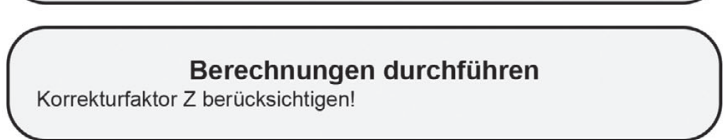

Abb. 2. Schema für den Ablauf einer Kalibrierung: Vor der eigentlichen Kalibrierung ist die Pipette zu identifizieren und an die Umgebungstemperatur im Prüfraum anzupassen.

\section{Berechnung des tatsächlichen Volumens}

Die Wägewerte sind nur die Massewerte des pipettierten Volumens. Eine Korrekturrechnung liefert das tatsächliche Volumen:

$$
V_{t \operatorname{Ref}}=W \cdot \frac{1}{\rho_{W}-\rho_{L}} \cdot\left(1-\frac{\rho_{L}}{\rho_{G}}\right) \cdot\left(1-\gamma \cdot\left(t-t_{\mathrm{Ref}}\right)\right)
$$

- $W=$ Wägewert,

- $V_{t \text { Ref }}=$ Volumen bei Referenztemperatur $\left(20^{\circ} \mathrm{C}\right)$,

- $t_{\text {Ref }}=$ Referenztemperatur $\left(20^{\circ} \mathrm{C}\right)$,

- $t=$ gemessene Temperatur,

- $\rho_{G}=$ Dichte des Justiergewichts,

- $\rho_{l}=$ Luftdichte in Abhängigkeit von Luftdruck, Temperatur und Luftfeuchte,

- $\rho_{W}=$ Wasserdichte in Abhängigkeit von der Temperatur,

- $\gamma=$ kubischer Ausdehnungskoeffizient.

Um die Berechnungen zu erleichtern, werden die verschiede- 


\begin{tabular}{rcc}
\hline $\begin{array}{l}\text { Volumenbereich } \\
{[\mu \mathrm{L}]}\end{array}$ & $\begin{array}{l}\text { Eintauchtiefe } \\
{[\mathrm{mm}]}\end{array}$ & $\begin{array}{l}\text { Wartezeit } \\
{[\mathrm{s}]}\end{array}$ \\
\hline $0,1-1$ & $1-2$ & 1 \\
$>1-100$ & $2-3$ & 1 \\
$>100-1000$ & $2-4$ & 1 \\
$>1000$ & $3-6$ & 3 \\
\hline
\end{tabular}

Tab. 1. Eintauchtiefe der Pipette und Wartezeit nach der Richtlinie DKD-R 8-1.

\begin{tabular}{rcc}
\hline $\begin{array}{l}\text { Volumenbereich } \\
{[\mu \mathrm{L}]}\end{array}$ & Waagenanzeige & Reproduzierbarkeit \\
\hline $0,1-10$ & 6-stellig & $\pm 0,003 \mathrm{mg}$ \\
$20-30$ & 5-stellig & $\pm 0,03 \mathrm{mg}$ \\
$50-250$ & 5-stellig & $\pm 0,02 \mathrm{mg}$ \\
$300-1000$ & 4-stellig & $\pm 0,1 \mathrm{mg}$ \\
\hline
\end{tabular}

Tab. 2. Geforderte Waagengenauigkeit nach ISO 8655 bei der Kalibrierung von Luftpolsterpipetten.

Weitere Berechnungen umfassen die Kalkulation des mittleren Volumens (V), des Variationskoeffizienten (VK) und der Richtigkeit (R).

$$
\begin{aligned}
& V=\bar{x} \cdot Z \\
& R[\%]=\frac{\bar{V}-V_{\text {Soll }}}{V_{\text {Soll }}} \cdot 100 \\
& V K[\%]=\frac{s \cdot 100}{\bar{V}}
\end{aligned}
$$

Die Werte für die Richtigkeit und den Variationskoeffizient werden dann mit den Fehlergrenzen der ISO 8655 verglichen. Nur Pipetten, die die Fehlergrenzen nicht überschreiten, dürfen eingesetzt werden.
Literatur

1) 1508655 , Volumenmessgeräte mit Hubkolben, Teil 1, 2, 6, Beuth-Verlag, Berlin, 2002.

2) Richtlinie DKD-R 8-1, „Kalibrierung von Kolbenhubpipetten mit Luftpolster", 2011. Download: http://delivr.com/2kvs2

3) K. H. Lochner, R. Felmann, J. Pfohl, DKD Expertenbericht DKD-E 8.2, „Analyse der Einflussgrößen auf die Kalibrierung von Kolbenhubpipetten mit Luftpolster", Braunschweig 2013, Download: http://delivr.com/2kvs2

Antonio Romaguera, Jahrgang 1964, studierte Biologie und promovierte 1991 am Institut für Mikrobiologie der Universität Hannover. Derzeit leitet er das Produktmanagement bei Brand in Werteim. Brand produziert neben Glas- und Volumenmessgeräten auch Geräte für die Life Sciences.

antonio.romaguera@brand.de

\section{Kurz notiert}

\section{TA Instruments kauft Messtechnikhersteller}

TA Instruments, eine Tochter des US-Geräteherstellers Waters, hat Scarabaeus Mess- und Produktionstechnik, Wetzlar, übernommen. Scarabaeus war bisher in Privatbesitz und produziert Geräte und Software für die Analyse von Kautschuk und die Kautschukverarbeitung. www.scarabaeus-gmbh.de

\section{Endress + Hauser übernimmt Analytik Jena}

Endress+Hauser, Hersteller von Prozessanalysatoren, übernimmt Analytik Jena. Das Unternehmen hält bereits $47 \%$ der Anteile an Analytik Jena und wird nun auch die restlichen Stimmrechte aufkaufen, unter anderem vom Land Thü- ringen. Der Angebotspreis beträgt 13,75 Euro pro Aktie.

Analytik Jena soll auch nach der Übernahme ein eigenständiges Unternehmen bleiben und den Standort Jena beibehalten.

delivr.com $/ 25 f k v$

\section{Bruker ins Mikroskopiegeschäft}

- Der Spektroskopehersteller Bruker hat einen Zulieferer von Produkten für die Fluoreszenzmikroskopie gekauft, das US-Unternehmen Prairie Technologies. Dieses setzte im letzten Jahr 11 Mio. Dollar um und beschäftigt 30 Mitarbeiter. Bruker erwirtschaftete mit 6000 Mitarbeitern im Jahr 2012 einen Umsatz von 1,8 Mrd. US-Dollar. Prairie Technologies wird Brukers Abteilung für Nanooberflächen erweitern.

ir.bruker.com

\section{Agilent trennt elektronische Messtechnik ab}

Agilent spaltet sich in zwei börsennotierte Unternehmen auf: Die Geschäftsbereiche Life Science, Diagnostik und Angewandte Märkte (LDA) behalten den Namen Agilent, das zweite Unternehmen umfasst Agilents Geschäft mit elektronischer Messtechnik. LDA erzielt als wachstumsstärkerer Sektor im Jahr 2013 einen geschätzten Umsatz von 3,9 Mrd. US-Dollar, eine Mrd. mehr als der Messtechnikbereich. Durch die Trennung soll Agilent vom Wachstum der LDABranche profitieren, unabhängig vom konjunkturempfindlichen Messtechnikmarkt. Die Transaktion soll Ende 2014 abgeschlossen sein.

delivr.com/2jt2f 